\title{
An Efficient Method for the Static Deflection Analysis of an Infinite Beam on a Nonlinear Elastic Foundation of One-Way Spring Model
}

\author{
Fayyaz ahmad, ${ }^{a} \quad$ Malik Zaka Ullah, ${ }^{b}$ Taek Soo Jang, ${ }^{c, *} \quad$ Eman S Alaidarous, ${ }^{b}$ \\ ${ }^{a}$ Dept. de Física i Enginyeria Nuclear, Universitat Politècnica de Catalunya, Comte d'Urgell 187, \\ 08036 Barcelona, Spain \\ ${ }^{b}$ Dept. of Mathematics, King Abdulaziz University, Jeddah 21589, Kingdom of Saudi Arabia \\ ${ }^{c}$ Dept. of Naval Architecture and Ocean Engineering, \\ Pusan National University,609-735, Busan, Republic of Korea
}

\begin{abstract}
An efficient numerical iterative method is constructed for the static deflection of an infinite beam on a nonlinear elastic foundation. The proposed iterative scheme consists of quasilinear method (QLM) and Green's function technique. The QLM translates the nonlinear ordinary differential equation into iterative linear ordinary differential equation. The successive iterations of quasilinear (QL) form of ODE show the quadratic convergence if an initial guess is chosen in the neighborhood of true solution. The Green's function technique converts the differential operator into an integral operator and the integral operator is approximated by discrete summation which finally gives us an iterative formula for the resulting set of algebraic equations. The numerical validity and efficiency is proved by simulating some nonlinear problems.
\end{abstract}

Keywords: Infinite beam; Non-linear deflection analysis; Non-linear elastic foundation; Transformed non-linear integral equation; Quasilinearization

\section{Introduction}

The accurate modeling of nonlinear deflection of an infinite beam on a nonlinear elastic foundation is important in practical engineering design application. It is always fascinating to find the closed form solution, some authors constructed closed-form solutions for the static and dynamic response of a uniform beam resting on a linear elastic foundation [1-3] and others proposed closed-form solution under the linearity assumption by using Green's function technique [4-7]. The static, dynamic and elastic stability analysis of a beam resting on a nonlinear elastic foundation was discussed by Beaufait and Hoadley [8], Massalas[9], Lakshmanan [10], and Hui [11], and by the same author and co-authors [12-15].

Jang et al. [16] used the Green's function technique to convert the non-linear ordinary differential equation for the non-linear deflection into nonlinear integral equation and translated it into system of nonlinear algebraic equations. By introducing a parameter, Jang constructed fixed-pint iterative formula for the solution of nonlinear algebraic equations. The author provided the convergence proof of his devised iterative scheme but did not discuss its order of convergence. Recently Park et al. [17] proposed an iterative method with the inclusion of

\footnotetext{
* Corresponding author: taek@pusan.ac.kr
}

pseudo spring coefficient but numerical experimentation shows that the rapid convergence of iterative scheme depends on the careful selection of pseudo spring coefficient and authors did not advise about the selection of parameter. In both articles, the authors constructed fixedpoint iterative methods. In the later author improved the convergence by the inclusion of a pseudo parameter. We also constructed a fixed-point iterative method based on the Newton-Raphson iterative method for the BVPs called the QLM which shows comparatively a better convergence relative to the work of $[16,17]$.

The Newton-Raphson is a quadratically convergent iterative method for a nonlinear algebraic or a system of nonlinear algebraic equations. The QLM is developed for nonlinear boundary value problem (BVP). Bellman and Kalaba [18] introduced QLM and later Mandelzweig and co-authors [19-21] provided the second order convergence proof for the BVPs. Recently many authors introduced higher order QLM methods for some specific BVPs and coupled BVPs [22-24]. The quadratic convergence of QLM provides fast convergence towards the true solution if we provide an initial guess in the neighborhood of solution curve [25]. Actually QLM is the generalization of Newton-Raphson method for BVPS. We used the QLM to construct the linear iterative form of governing nonlinear fourth-order ordinary differential equation and finally the Green's function method application to QL iterative form of nonlinear differential 
equations provides a system of nonlinear algebraic equations. It is also noted that the selection of parameter is not crucial for the rapid convergence in the newly devised numerical iterative scheme.

\section{Mathematical Modeling}

The linear deflection of an infinite beam on an elastic foundation can be modeled by the fourth-order differential equation (the weight of the beam is not included):

$$
E I \frac{d^{4} u}{d x^{4}}+f(u)=w(x),
$$

and the reaction force is given as

$$
f(u)=k u+N(u)
$$

where $E, I, K, N(u)$ and $w(x)$ are Young's modulus, the mass moment of inertia, a linear spring coefficient, a nonlinear part of spring force, and external load respectively. The boundary conditions are provided as

$$
u, \frac{d u}{d x}, \frac{d^{2} u}{d x^{2}}, \text { and } \frac{d^{3} u}{d x^{3}} \rightarrow 0 \text { as }|x| \rightarrow \infty .
$$

The closed-form solution [4-7] of (1) by neglecting the nonlinear part $N(u)$ in $(2)$ is

$$
u(x)=\frac{1}{E I} \int_{-\infty}^{\infty} G(x, \xi ; k) w(\xi) d \xi
$$

where Green's function $G$ is defined as

$$
\begin{aligned}
& G(x, \xi ; k)=\frac{\alpha}{2 k} e^{-\alpha|\xi-x| / \sqrt{2}} \sin \left(\frac{\alpha|\xi-x|}{\sqrt{2}}+\frac{\pi}{4}\right), \\
& \alpha=\sqrt[4]{k / E I}
\end{aligned}
$$

where loading condition is localized i.e. $|w(x)| \rightarrow 0$ as $|x| \rightarrow \infty$. In the present study we combine two methods quasilinear method and Green's function technique. The QL form of (1) is written as

$$
E I \frac{d^{4} u_{n+1}}{d x^{4}}+\frac{d f\left(u_{n}\right)}{d u} u_{n+1}=\frac{d f\left(u_{n}\right)}{d u} u_{n}-f\left(u_{n}\right)+w(x) .
$$

By adding $k_{p}$ pseudo spring coefficient in both sides of (6), we obtain

$$
\begin{aligned}
& \frac{d^{4} u_{n+1}}{d x^{4}}+\frac{k_{p}}{E I} u_{n+1}=\frac{1}{E I}\left[k_{p} u_{n+1}+\frac{d f\left(u_{n}\right)}{d u} u_{n}\right. \\
& \left.-\frac{d f\left(u_{n}\right)}{d u} u_{n+1}-f\left(u_{n}\right)+w(x)\right] .
\end{aligned}
$$

The Green's functions solution of (6) is

$$
\begin{aligned}
& u_{n+1}=\frac{1}{E I} \int_{-\infty}^{\infty} G\left(x, \xi ; k_{p}\right)\left[\left(k_{p}-\frac{d f\left(u_{n}\right)}{d u}\right) u_{n+1}\right. \\
& \left.+\frac{d f\left(u_{n}\right)}{d u} u_{n}-f\left(u_{n}\right)+w(\xi)\right] d \xi
\end{aligned}
$$

We select a positive number $r$ such that $u(x) \approx 0$ and $w(x) \approx 0$ if $x \notin[-r, r]$. Suppose the discretization of interval $[-r, r]$ is $\left\{x_{1}, x_{2}, \cdots, x_{n_{x}}\right\}$ then (8) can be expressed in this way

$$
\begin{aligned}
& u\left(x_{i}\right)_{n+1}=\frac{1}{E I} \sum_{j=1}^{n_{x}} G\left(x_{i}, x_{j} ; k\right) b(j)\left[\left(k_{p}-\frac{d f\left(u\left(x_{j}\right)_{n}\right)}{d u}\right)\right. \\
& \left.u\left(x_{j}\right)_{n+1}+\frac{d f\left(u\left(x_{j}\right)_{n}\right)}{d u} u\left(x_{j}\right)_{n}-f\left(u\left(x_{j}\right)_{n}\right)+w\left(x_{j}\right)\right] \\
& \text { for } i=1,2, \cdots, n_{x} .
\end{aligned}
$$

By defining the diagonal matrix of a vector $\mathbf{v}=$ $\left[v_{1}, v_{2}, \cdots, v_{n_{x}}\right]^{T}$

$$
\operatorname{diag}(\mathbf{v})=\left(\begin{array}{ccccc}
v_{1} & 0 & 0 & \cdots & 0 \\
0 & v_{2} & 0 & \cdots & 0 \\
\vdots & \vdots & \vdots & \cdots & \vdots \\
0 & 0 & 0 & \cdots & v_{n_{x}}
\end{array}\right)
$$

We get the vectorial form of (9) (named as Fayyaz's iterative method (FIM))

$$
\begin{aligned}
& \boldsymbol{u}_{\boldsymbol{n + 1}}+\boldsymbol{G} \operatorname{diag}(\boldsymbol{b})\left(\operatorname{diag}\left(f^{\prime}\left(\boldsymbol{u}_{\boldsymbol{n}}\right)\right)-k_{p} \boldsymbol{I}_{n_{n_{x}} \times n_{n_{x}}}\right) \boldsymbol{u}_{\boldsymbol{n + 1}} \\
& =\boldsymbol{G} \operatorname{diag}(\boldsymbol{b})\left[\operatorname{diag}\left(f^{\prime}\left(\boldsymbol{u}_{\boldsymbol{n}}\right)\right) \boldsymbol{u}_{\boldsymbol{n}}-f\left(\boldsymbol{u}_{\boldsymbol{n}}\right)+\boldsymbol{w}\right], \\
& {\left[\boldsymbol{I}_{n_{n_{x}} \times n_{n_{x}}}+\boldsymbol{G} \operatorname{diag}(\boldsymbol{b})\left(\operatorname{diag}\left(f^{\prime}\left(\boldsymbol{u}_{\boldsymbol{n}}\right)\right)-k_{p} \boldsymbol{I}_{n_{n_{x}} \times n_{n_{x}}}\right)\right]} \\
& \boldsymbol{u}_{\boldsymbol{n}+\mathbf{1}}=\boldsymbol{G} \operatorname{diag}(\boldsymbol{b})\left[\operatorname{diag}\left(f^{\prime}\left(\boldsymbol{u}_{\boldsymbol{n}}\right)\right) \boldsymbol{u}_{\boldsymbol{n}}-f\left(\boldsymbol{u}_{\boldsymbol{n}}\right)+\boldsymbol{w}\right], \\
& \boldsymbol{u}_{\boldsymbol{n + 1}}=\left[\boldsymbol{I}_{n_{n_{x}} \times n_{n_{x}}}+\boldsymbol{G} \operatorname{diag}(\boldsymbol{b})\left(\operatorname{diag}\left(f^{\prime}\left(\boldsymbol{u}_{\boldsymbol{n}}\right)\right)-k_{p}\right.\right. \\
& \left.\left.\boldsymbol{I}_{n_{n_{x}} \times n_{n_{x}}}\right)\right]^{-1} \boldsymbol{G} \operatorname{diag}(\boldsymbol{b})\left[\operatorname{diag}\left(f^{\prime}\left(\boldsymbol{u}_{\boldsymbol{n}}\right)\right) \boldsymbol{u}_{\boldsymbol{n}}-f\left(\boldsymbol{u}_{\boldsymbol{n}}\right)+\boldsymbol{w}\right] \text {, }
\end{aligned}
$$

where

$\boldsymbol{G}=\frac{1}{E I}\left(\begin{array}{ccc}G\left(x_{1}, x_{1} ; k_{p}\right) & \cdots & G\left(x_{1}, x_{n_{x}} ; k_{p}\right) \\ G\left(x_{2}, x_{1} ; k_{p}\right) & \cdots & G\left(x_{2}, x_{n_{x}} ; k_{p}\right) \\ \vdots & \cdots & \vdots \\ G\left(x_{n_{x}}, x_{1} ; k_{p}\right) & \cdots & G\left(x_{n_{x}}, x_{n_{x}} ; k_{p}\right)\end{array}\right)$,
$\boldsymbol{u}_{\boldsymbol{n}}=\left(\begin{array}{c}\left(u\left(x_{1}\right)\right)_{n} \\ \left(u\left(x_{2}\right)\right)_{n} \\ \vdots \\ \left(u\left(x_{n_{x}}\right)\right)_{n}\end{array}\right), \quad \boldsymbol{I}_{n_{n_{x}} \times n_{n_{x}}}=\left(\begin{array}{ccccc}1 & 0 & 0 & \cdots & 0 \\ 0 & 1 & 0 & \cdots & 0 \\ \vdots & \vdots & \vdots & \cdots & \vdots \\ 0 & 0 & 0 & \cdots & 1\end{array}\right)$,

and $\boldsymbol{b}$ is a weight vector to convert integral into summation e.g. in the case of trapezoidal-rule the weight vector $\boldsymbol{b}=h[1 / 2,1,1, \cdots, 1,1 / 2]$ ( $h$ is uniform step size $)$.

\section{Numerical Results}

For the numerical results comparison, we listed here the iterative scheme proposed in [17] and we called it Jang's iterative method (JIM). The values of parameters are taken from [17].

$$
\boldsymbol{u}_{\boldsymbol{n}+\mathbf{1}}=\boldsymbol{G} \operatorname{diag}(\boldsymbol{b})\left[k_{p} \boldsymbol{u}_{\boldsymbol{n}}-f\left(\boldsymbol{u}_{\boldsymbol{n}}\right)+\boldsymbol{w}\right] .
$$


We define the relative error as follows:

$$
\begin{aligned}
& \operatorname{Error}(n)=\frac{\left\|u(x)-u_{n}(x)\right\|_{2}}{\|u(x)\|_{2}}, \\
& \text { where }\|z\|_{2}=\left(\sum_{i=0}^{n_{x}}\left|z_{i}\right|^{2}\right)^{1 / 2} .
\end{aligned}
$$

In the present study, we assume the spring force of nonlinear elastic foundation obeys the definition.

$$
f(u)= \begin{cases}k u+\gamma u^{m}, & \text { if } u \geq 0 \\ 0, & \text { if } u \leq 0\end{cases}
$$

In Table 1, three test function are selected and there corresponding loading functions are also depicted. The analytical solution $u(x)$ and loading function $w(x)$ are shown in Figure 1. In Figures 2, 3, 4, the convergence of iterative schemes FIM and JIM are shown for three cases (a), (b) and (c). For the case(a) our proposed iterative scheme converges in three iterations and order of absolute error is $O\left(10^{-6}\right)$ which is clearly superior than absolute error order for the scheme JIM. Similarly for other two cases in two iterations the scheme FIM achieved the absolute error of order $O\left(10^{-6}\right)$. In Table 2, We compared the relative absolute error of schemes JIM and FIM against the different combinations of parameters for the designed three study cases. We fixed the values of different parameters for three problems and then calculate the order of absolute relative error for both schemes. The different combinations of linear and nonlinear coefficients for the fixed number of iterations show the superiority of our proposed scheme. Even by including high nonlinearity in the form of power law, results depict the fast convergence with good accuracy for our iterative scheme FIM.

It is also interesting to test the accuracy and speed of convergence for both iterative schemes for discontinuous loading function $w(x)$ against different values of parameter $k_{p}$ and $k$. The rectangular loading function $w_{1}(x)$ and $w_{2}(x)$ are taken from [17]. In Figure 5 and 6 we depicted both discontinuous loading functions. For the discontinuous load function $w_{1}(x)$, we keep $k=1$ fix and check the convergence for three different values of $k_{p} \in\{3,6,10\}$. For each case a sequence of six iterations is plotted in Figure 5 which clearly describe the convergence of our proposed iterative scheme. For the second case we define $w_{2}(x)$ with multiple discontinuities and keep $k_{p}=3$ fix and plotted the results for different values of $k \in\{1,1.5,2\}$ where rapid convergence toward true solution can be observed in Figure 6. Note that for the discontinuous loading function, we do not have analytical solution to calculate the order of absolute error.

$$
\begin{aligned}
& w_{1}(x)= \begin{cases}1 & |x| \leq 1 \\
0 & \text { otherwise }\end{cases} \\
& w_{2}(x)= \begin{cases}1 & 1 \leq|x| \leq 2,4 \leq|x| \leq 5 \\
0 & \text { otherwise }\end{cases}
\end{aligned}
$$

Figures 5, 6 show the fast convergence of iterative scheme FIM and iterative scheme JIM.

Table 1. Three cases of exact solution and loading function

\begin{tabular}{lll}
\hline \hline \multicolumn{2}{l}{ Cases $u(x)$} & $w(x)$ \\
\hline \hline $\mathrm{a}$ & $e^{-x^{2}}$ & $\gamma e^{-3 x^{2}}+k e^{-x^{2}}+E I\left(12 e^{-x^{2}}-\right.$ \\
& & $\left.48 x^{2} e^{-x^{2}}+16 x^{4} e^{-x^{2}}\right)$ \\
$\mathrm{b} \quad \sin (x) e^{-x^{2}}$ & $k \sin (x) e^{-x^{2}}+\gamma \sin (x)^{3} e^{-3 x^{2}}+$ \\
& & $E I\left(25 \sin (x) e^{-x^{2}}+56 x \cos (x) e^{-x^{2}}-\right.$ \\
& & $32 x^{3} \cos (x) e^{-x^{2}}-72 x^{2} \sin (x) e^{-x^{2}}+$ \\
& & \\
$\mathrm{c}$ & $\sin (x) e^{4} \sin (x) e^{-x^{2} / 4}$ & $k \sin (x) e^{-x^{2} / 4}+\gamma \sin (x)^{3} e^{-3 x^{2} / 4}$ \\
& & $E I\left(19 \sin (x) / 4 e^{-x^{2} / 4}+5 x \cos (x)\right.$ \\
& & $e^{-x^{2} / 4}-x^{3} \cos (x) / 2 e^{-x^{2} / 4}-$ \\
& $9 x^{2} \sin (x) / 4 e^{-x^{2} / 4}$ \\
& $\left.x^{4} \sin (x) / 16 e^{-x^{2} / 4}\right)$ \\
&
\end{tabular}

Table 2. Simulation parameter: $n_{x}=200, E=1, I=1$, $u_{0}=0$

\begin{tabular}{llllllll}
\hline \hline Probs & iter & $k_{p}$ & $k$ & $\gamma$ & $m$ & $\begin{array}{l}\text { Error }(n) \\
\text { JIM }\end{array}$ & $\begin{array}{l}\text { Error }(n) \\
\text { FIM }\end{array}$ \\
\hline $\mathrm{a}$ & 5 & 0.1 & 0.1 & 0.2 & 3 & 0.69927 & $6.5488 \mathrm{e}-007$ \\
$\mathrm{~b}$ & 5 & 0.1 & 0.1 & 0.2 & 3 & $2.4983 \mathrm{e}-006$ & $2.4984 \mathrm{e}-006$ \\
$\mathrm{c}$ & 5 & 0.1 & 0.1 & 0.2 & 3 & 0.00095859 & $1.964 \mathrm{e}-005$ \\
\hline $\mathrm{a}$ & 5 & 0.5 & 0.1 & 0.2 & 3 & 0.037064 & $6.5988 \mathrm{e}-007$ \\
$\mathrm{~b}$ & 5 & 0.5 & 0.1 & 0.2 & 3 & 0.042426 & $2.5213 \mathrm{e}-006$ \\
$\mathrm{c}$ & 5 & 0.5 & 0.1 & 0.2 & 3 & 0.050846 & $1.0096 \mathrm{e}-005$ \\
\hline $\mathrm{a}$ & 5 & 1 & 1 & 2 & 4 & 0.79909 & $2.858 \mathrm{e}-005$ \\
$\mathrm{~b}$ & 5 & 1 & 1 & 2 & 4 & $4.0865 \mathrm{e}-006$ & $2.5446 \mathrm{e}-006$ \\
$\mathrm{c}$ & 5 & 1 & 1 & 2 & 4 & 0.062785 & $2.9007 \mathrm{e}-006$ \\
\hline $\mathrm{a}$ & 7 & 1 & 1 & 2 & 10 & 0.51141 & $6.0644 \mathrm{e}-007$ \\
$\mathrm{~b}$ & 7 & 1 & 1 & 2 & 10 & $2.522 \mathrm{e}-006$ & $2.5264 \mathrm{e}-006$ \\
$\mathrm{c}$ & 7 & 1 & 1 & 2 & 10 & $2.5667 \mathrm{e}-006$ & $2.5744 \mathrm{e}-006$ \\
\hline $\mathrm{a}$ & 5 & 1 & 2 & 2 & 7 & 1.3661 & $3.005 \mathrm{e}-006$ \\
$\mathrm{~b}$ & 5 & 1 & 2 & 2 & 7 & 0.18156 & $2.4972 \mathrm{e}-006$ \\
$\mathrm{c}$ & 5 & 1 & 2 & 2 & 7 & 0.59131 & $1.7652 \mathrm{e}-006$ \\
\hline \hline
\end{tabular}

\section{Conclusion}

In this study, we validate our newly proposed iterative scheme which is the combination of two method namely QLM and Green's function method. The role of QLM is to construct an iterative scheme by linearizing nonlinear terms and Green's function method converts the linearized differential equation into integral equation, and it is an easy task to convert system of integral equations into a system of nonlinear algebraic equations by using any quadrature rule. The computational cost of our proposed iterative scheme is higher than iterative scheme 

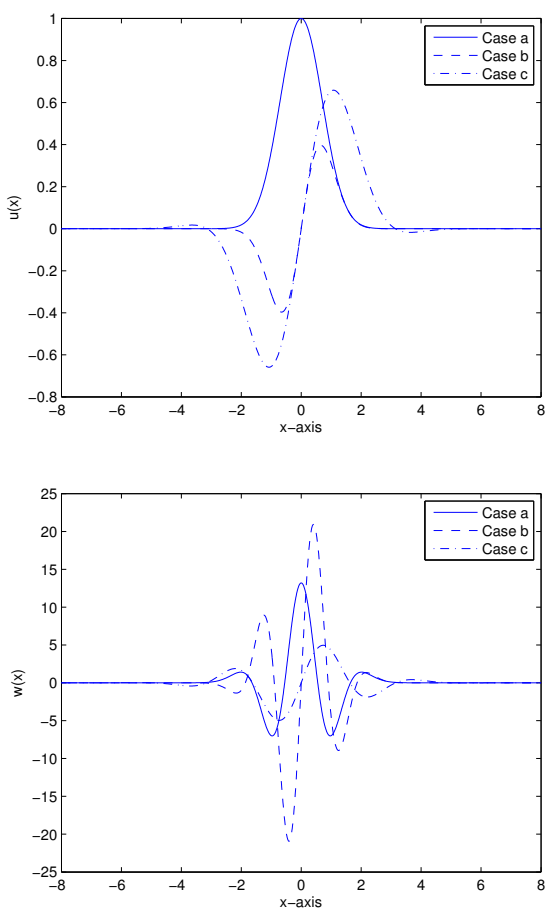

Fig. 1. Three cases of exact solution and loading function in Table 1

JIM because "in each step" the calculation of inverse of a matrix is involved which is an expensive operation but the convergence is much faster. we achieved accuracy of magnitude $10^{-6}$ almost in three or two iterations. On the other hand, the iterative scheme JIM does not require the calculation of any inverse of a matrix which depends on the proper selection of parameter $k_{p}$ but the value of $k_{p}$ does not much affect the convergence speed of our proposed iterative method.

\section{References}

[1] M. Hetenyi, Beams on Elastic Foundation, The University of Michigan Press, Ann Arbor, Mich, USA, 1946.

[2] C. Miranda and K. Nair, "Finite beams on elastic foundation," Journal of the Structural Division, vol. 92, pp. 131-142, 1966.

[3] B. Y. Ting, "Finite beams on elastic foundation with restraints," Journal of the Structural Division, vol. 108, no. 3, pp. 611-621, 1982.

[4] S. Timoshenko, "Method of analysis of statistical and dynamical stress in rail," in Proceeding of the International Congress of Applied Mechanics, pp. 407-418, Zurich, Switzerland, 1926.

[5] J. T. Kenney,"Steady-state vibrations of beam on elastic foundation for moving load," Journal of Applied Mechanics, vol. 21, pp. 359-364, 1954.

[6] H. Saito and T. Murakami, "Vibrations of an infinite beam on an elastic foundation with consideration of mass of a foundation," The Japan Society of Mechanical Engineers, vol. 12, pp. 200-205, 1969.

[7] L. Fryba, "Infinitely long beam on elastic foundation under moving load," Aplikace Matematiky, vol. 2, pp. 105-132, 1957.

[8] F. W. Beaufait and P. W. Hoadley, "Analysis of elastic beams on nonlinear foundations," Computers \& Structures, vol. 12, no. 5, pp. 669-676, 1980.

[9] C. Massalas, "Fundamental frequency of vibration of a beam on a non-linear elastic foundation," Journal of Sound and Vibration, vol. 54, no. 4, pp. 613-615, 1977.

[10] M. Lakshmanan, "Comment on the fundamental frequency of vibration of a beam on a non-linear elastic foundation," Journal of Sound and Vibration, vol. 58, no. 3, pp. 455-457, 1978.

[11] D. Hui, "Postbuckling behavior of infinite beams on elastic foundations using Koiter's improved theory," International Journal of Non-Linear Mechanics, vol. 23, no. 2, pp. 113-123, 1988.

[12] T.S. Jang, H.G. Sung, "A new semi-analytical method for the non-linear static analysis of an infinite beam on a non-linear elastic foundation: a general approach to a variable beam cross-section", International Journal of Non-linear Mechanics 2012 (47) 132-139.

[13] S.W. Choi, T.S. Jang, "Existence and uniqueness of nonlinear deflections of an infinite beam resting on a non-uniform non-linear elastic foundation", Boundary Value Problems 2012(2012) DOI:10.1186/1687-2770-2012-5.

[14] T.S. Jang, "A new semi-analytical approach to large deflections of Bernoulli-Euler-v. Karman beams on a linear elastic foundation: nonlinear analysis of infinite beams", International Journal of Mechanical Sciences 2013 (66) 22-32 .

[15] T.S. Jang, " A general method for analyzing moderately large deflections of a non-uniform beam: an infinite BernoulliEuler-von Karman beam on a non-linear elastic foundation", Acta Mechanica, DOI 10.1007/s00707-013-1077-x

[16] T.S. Jang, H.S Baek, J.K. Paik, "A new method for the non-linear deflection analysis of an infinite beam resting on a non-linear elastic foundation," International Journal of NonLinear Mechanics 46 (2011) 339-346.

[17] Jinsoo Park, Hyeree Bai, and T. S. Jang, "A Numerical Approach to Static Deflection Analysis of an Infinite Beam on a Nonlinear Elastic Foundation: One-Way Spring Model," Journal of Applied Mathematics, Volume 2013, Article ID 136358, 10 pages, http://dx.doi.org/10.1155/2013/136358.

[18] R. E. Bellman and R. E. Kalaba, "Quasilinearization and Nonlinear Boundary-Value Problems", Elsevier Publishing Company, New York,1965.

[19] V. B. Mandelzweig, F. Tabakin, "Quasilinearization Approach to Nonlinear Problems in Physics with Application to Nonlinear ODEs," http://arxiv.org/pdf/physics/0102041.pdf

[20] V. B. Mandelzweig, "Quasilinearization method and its verification on exactly solvable models in quantum mechanics," J. Math. Phys. 40, 6266 (1999).

[21] R. Krivec and V. B. Mandelzweig, "Numerical investigation of quasilinearization method in quantum mechanics," Computer Physics Communications, 138, 69 (2001).

[22] R. Kalaba, "On nonlinear differential equations, the maximum operation and monotone convergence," J. Math. Mech. 8, 519 (1959).

[23] Sandile S. Motsa, Precious Sibanda, Some modification of the quasilinearization method with higher-order convergence for solving nonlinear BVPs, J. Numer. Algor., DOI 10.1007/s11075-012-9629-z.

[24] Eman S. Alaidarous, Malik Zaka Ullah, Fayyaz Ahmad, and A.S. Al-Fhaid, An Efficient Higher-Order Quasilinearization Method for Solving Nonlinear BVPs, Journal of Applied Mathematics, Volume 2013 (2013), Article ID 259371, 11 pages, http://dx.doi.org/10.1155/2013/259371

[25] S.Cuomo,A. Marasco, A numerical approach to nonlinear two-point boundary value problems for ODEs, Computers and Mathematics with Applications Volume 55, Issue 11, June 2008, Pages 2476-2489. 

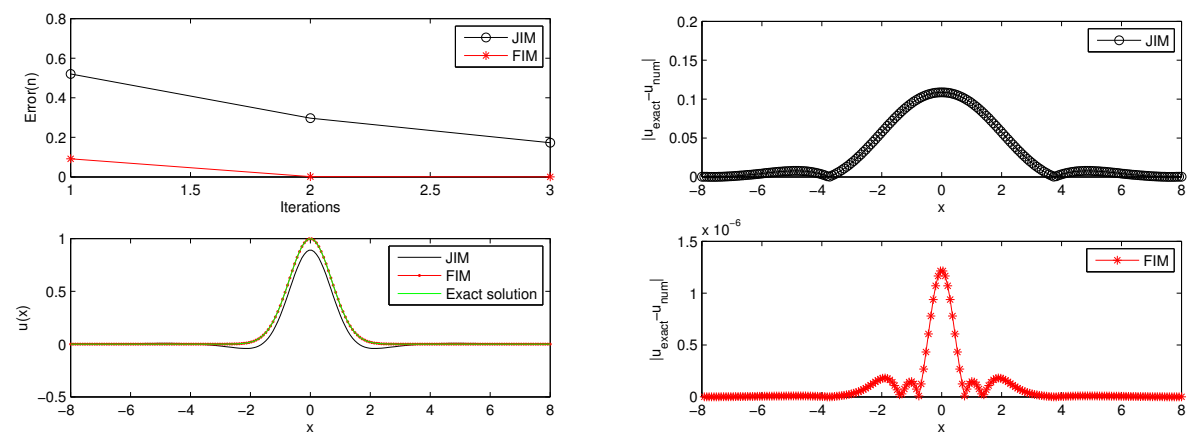

Fig. 2. Case (a), Numerical solution comparison $\left(E=I=1, k=1, k_{p}=3, \gamma=0.2, m=3\right.$ initial guess $u_{0}=0$, number of iterations $=3$, trapezoidal integration, number of grid points $n_{x}=200$ )
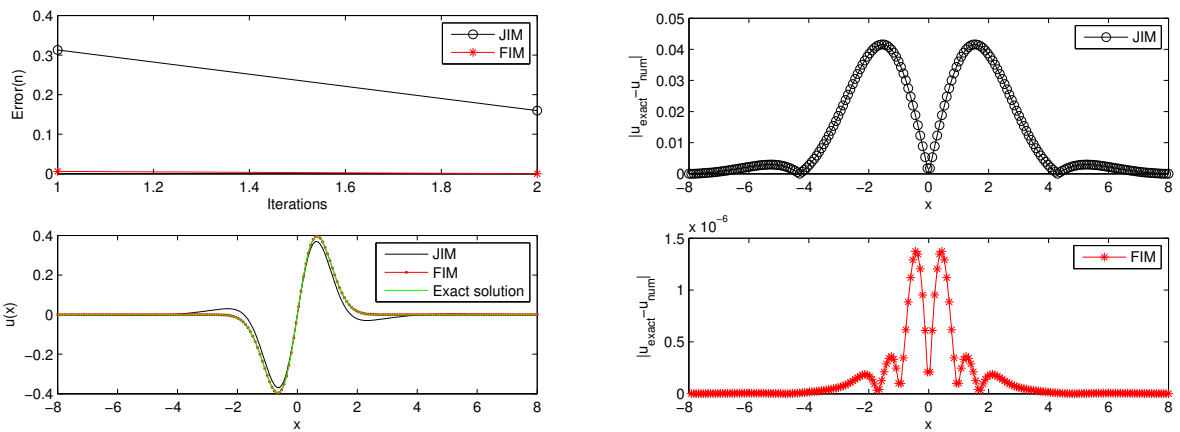

Fig. 3. Case (b), Numerical solution comparison $\left(E=I=1, k=1, k_{p}=3, \gamma=0.2, m=3\right.$, initial guess $u_{0}=0$, number of iterations $=2$, trapezoidal integration, number of grid points $n_{x}=200$ )
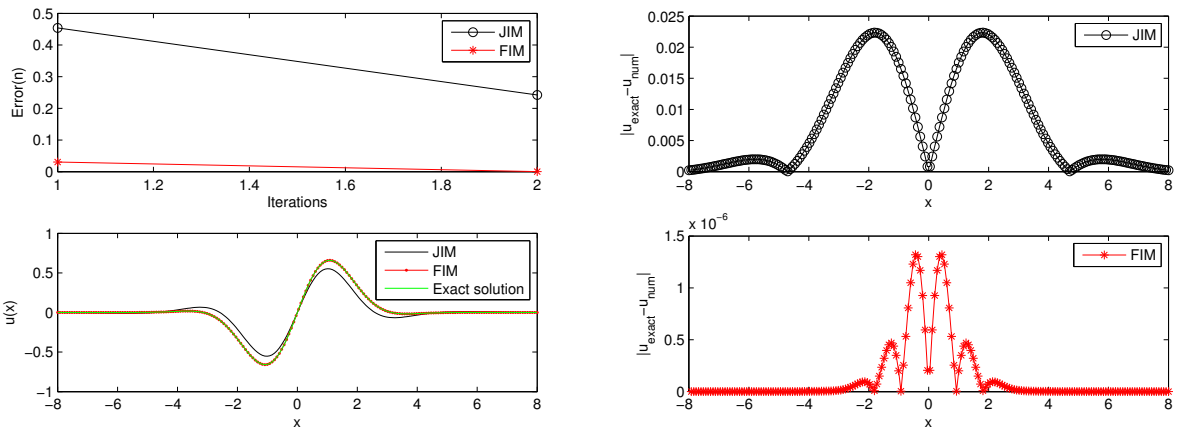

Fig. 4. Case (c), Numerical solution comparison $\left(E=I=1, k=1, k_{p}=3, \gamma=0.2, m=3\right.$, initial guess $u_{0}=0$, number of iterations $=2$, trapezoidal integration, number of grid points $n_{x}=200$ ) 


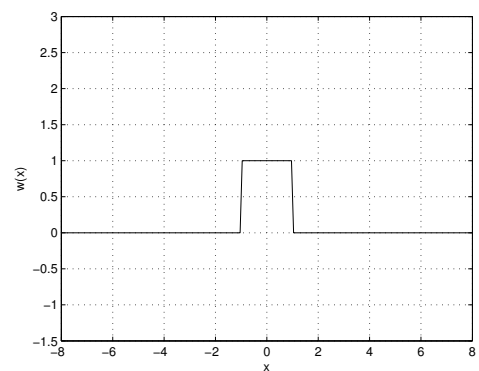

(i) Loading function
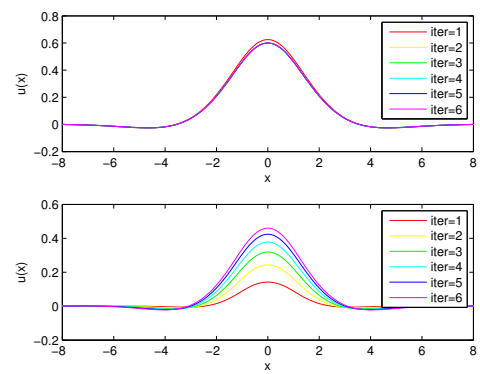

(iii) $\operatorname{case}(\mathrm{b}) k_{p}=6$
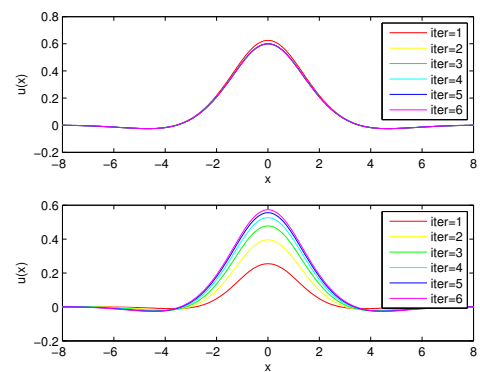

(ii) $\operatorname{case}(\mathrm{a}) k_{p}=3$
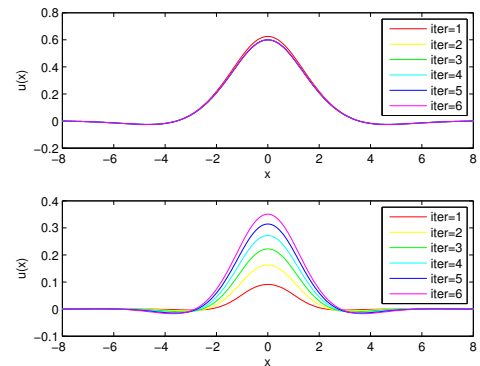

(iv) $\operatorname{case}(\mathrm{c}) k_{p}=10$

Fig. 5. Numerical solution comparison between FIM (top) and JIM (bottom) $(E=I=1, k=1, \gamma=0.2, m=3$, initial guess $u_{0}=0$, number of iterations $=6$, trapezoidal integration, number of grid points $n_{x}=200$ )

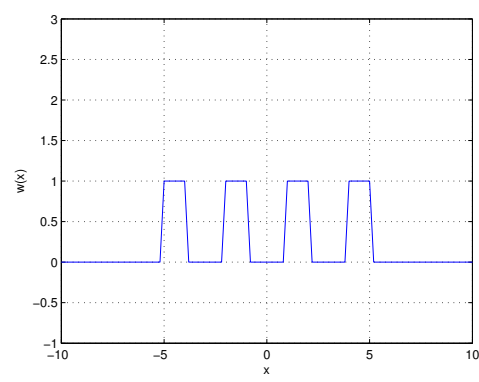

(i) Loading function
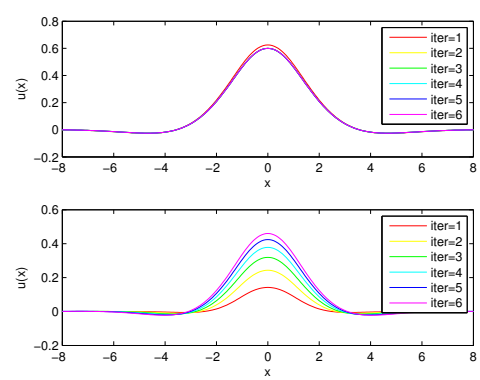

(iii) $\operatorname{case}(\mathrm{b}) k=1.5$
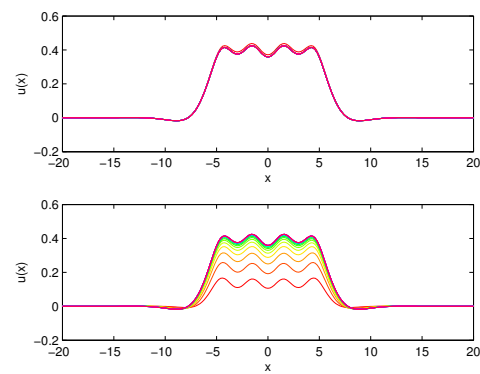

(ii) $\operatorname{case}(\mathrm{a}) k=1$
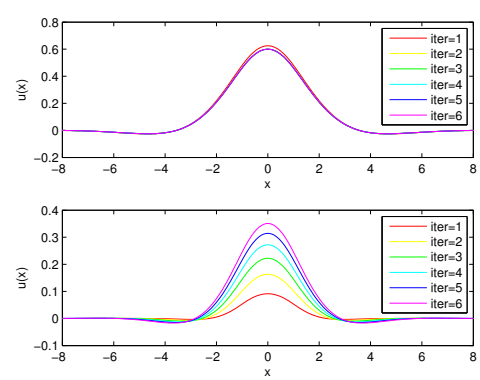

(iv) $\operatorname{case}(\mathrm{c}) k=2$

Fig. 6. Numerical solution comparison between FIM (top) and JIM (bottom) $\left(E=I=1, k_{p}=3, \gamma=0.2, m=3\right.$, initial guess $u_{0}=0$, number of iterations $=20$, trapezoidal integration, number of grid points $\left.n_{x}=200\right)$ 